\title{
Panorama da saúde mental da enfermagem durante a COVID-19 no Brasil: uma revisão integrativa
}

\author{
An overview of the mental health of nurses during COVID-19 in Brazil: an \\ integrative review
}

\begin{abstract}
Una visión general de la salud mental de las enfermeras durante el COVID-19 en Brasil: una revisión integradora
\end{abstract}

Alyci Christini Reis Ferreira ${ }^{1 *}$, Francisco Railson Bispo de Barros².

\begin{abstract}
RESUMO
Objetivo: Identificar a repercussão da pandemia do novo coronavírus (COVID-19) na saúde mental da enfermagem brasileira que atua na linha de frente do cuidado. Métodos: Trata-se de uma Revisão Integrativa da Literatura (RIL) realizada no mês de junho de 2021, mediante consulta as bases de dados BDENF, LILACS, PUBMED e SCIELO. Resultados: Dentre os onze artigos que foram incluídos na amostra, 4 foram da LILACS, 4 da SCIELO e 3 da PUBMED, os quais destacam os efeitos psicológicos da pandemia e a prevalência de níveis mais altos de sofrimento mental entre os profissionais de enfermagem na linha de frente do cuidado, assim como as estratégias adotadas para o combate ao estresse emocional. Considerações finais: Diante do cenário de calamidade vivido durante a pandemia de COVID-19, os enfermeiros foram bastante afetados quanto à sua saúde mental. Incentivos públicos como bom treinamento de uso de EPIs, oferta de terapia por profissionais de saúde mental e aumento de remuneração fizeram com que houvesse uma diminuição no quadro de transtornos mentais sofridos por esses profissionais.
\end{abstract}

Palavras-chave: COVID-19, Enfermagem, Saúde mental.

\begin{abstract}
Objective: To identify the impact of the new coronavirus pandemic (COVID-19) on the mental health of Brazilian nursing staff who work on the front line of care. Methods: This is an Integrative Literature Review (RIL) carried out in June 2021, by consulting the BDENF, LILACS, PUBMED and SCIELO databases. Results: Among the eleven articles that were included in the sample, 4 were from LILACS, 4 from SCIELO and 3 from PUBMED, which highlight the psychological effects of the pandemic and the prevalence of higher levels of mental suffering among nursing professionals in the line front of care, as well as the strategies adopted to combat emotional stress. Final considerations: Faced with the calamity scenario experienced during the COVID-19 pandemic, nurses were greatly affected in terms of their mental health. Public incentives such as good training in the use of PPE, the offer of therapy by mental health professionals and an increase in remuneration meant that there was a reduction in the mental disorders suffered by these professionals.
\end{abstract}

Key words: COVID-19, Nursing, Mental health.

\section{RESUMEN}

Objetivo: Identificar el impacto de la nueva pandemia de coronavirus (COVID-19) en la salud mental del personal de enfermería brasileño que trabaja en la primera línea de atención. Métodos: Se trata de una

${ }^{1}$ Centro Universitário do Norte (UNINORTE), Manaus - AM. *E-mail: alyci.christini@gmail.com

2 Universidade do Estado do Amazonas (UEA), Manaus - AM.

SUBMETIDO EM: 9/2021

ACEITO EM: 9/2021

PUBLICADO EM: 9/2021 
Revisión de Literatura Integrativa (RIL) realizada en junio de 2021, mediante la consulta de las bases de datos BDENF, LILACS, PUBMED y SCIELO. Resultados: De los once artículos que se incluyeron en la muestra, 4 fueron de LILACS, 4 de SCIELO y 3 de PUBMED, que destacan los efectos psicológicos de la pandemia y la prevalencia de mayores niveles de sufrimiento mental entre los profesionales de enfermería en el frente de línea. del cuidado, así como las estrategias adoptadas para combatir el estrés emocional. Consideraciones finales: Ante el escenario de calamidad vivido durante la pandemia de COVID-19, las enfermeras se vieron muy afectadas en términos de su salud mental. Los incentivos públicos como la buena formación en el uso de EPI, la oferta de terapias por parte de los profesionales de la salud mental y el aumento de la retribución supusieron una reducción de los trastornos mentales que padecían estos profesionales.

Palabras clave: COVID-19, Enfermería, Salud mental.

\section{INTRODUÇÃO}

Os primeiros casos da Coronavirus Disease of 2019 (COVID-19) causada pelo Severe acute respiratory syndrome coronavirus 2 (SARS-CoV-2) foram detectados em 2019 na cidade de Wuhan, China Central (HUANG C, et al., 2020). Os infectados com o vírus apresentam desde sintomas mais leves aos mais severos, e uma boa parcela da população pode não apresentar sintomas, caracterizando-se como assintomáticos (WANG D, et al., 2020). Os sintomas mais comuns de pacientes infectados são: febre alta, tosse frequente e dificuldade para respirar, além de, em pacientes com quadros de doenças respiratórias já presentes, os sintomas associados ao sistema respiratório se agravam (CIOTTI M, et al., 2020; WANG D, et al., 2020; ZHU N, et al., 2020).

Além disso, a longo prazo, os pacientes infectados podem apresentar complicações também entéricas, hepáticas e neurológicas, e, devido às complicações no sistema respiratório, a doença apresenta altas taxas de internação em Unidade de Terapia Intensiva (UTI) e de mortalidade. A infecção por SARS-CoV-2 pode ocorrer tanto no sistema respiratório superior quanto no inferior, dessa forma, pode ser transmitida de forma direta ou indireta apresentando, por isso, uma alta taxa de transmissão (HUANG C, et al., 2020).

No Brasil, de acordo com os dados epidemiológicos do Ministério da Saúde (MS) os casos de COVID-19 cresceram de forma acelerada. Em janeiro de 2020 eram apenas 2.798 casos registrados, já em junho de 2020 os casos saltaram para 850.514 casos, com 41.828 óbitos registrados. Fechando o ano de 2020 , o Brasil registrou 7.465.806 casos de COVID-19 contabilizados e mais de 190.000 mortes. Fevereiro de 2021 apresentou 10.517.232 casos, com 254.221 óbitos contabilizados e, em julho de 2021, os casos já somavam mais de 20 milhões, com mais 500 mil óbitos decorrentes de COVID-19 (MINISTÉRIO DA SAÚDE, 2021).

O aumento de casos desse vírus colapsou o sistema de saúde brasileiro, onde havia hospitais com 100\% de sua capacidade e profissionais da saúde trabalhando cerca de 48 horas seguidas para sanar as dificuldades enfrentadas. Nesse cenário de caos e calamidade apresentado por conta da pandemia de COVID-19, os profissionais que mais sofrem com a alta taxa de infecção e contaminação são os profissionais da saúde, uma vez que estão atuando na linha de frente e auxiliando no tratamento de pacientes infectados que chegam aos hospitais (LAl J, et al., 2020).

Por conta do medo constante de exposição direta, as incertezas acerca do vírus e a alta taxa de mortalidade por conta do COVID-19, vários profissionais da saúde passaram a desenvolver doenças mentais como, medo, ansiedade, depressão, angústia, insônia, extremo cansaço mental, dentre outros sintomas que afetam a saúde mental (PRADO AD, et al. 2020).

Dentre os profissionais de saúde que atuaram na linha de frente durante esta pandemia, estima-se que os mais afetados são enfermeiras e mulheres (LAI J, et al., 2020). Como estes profissionais são os que mais auxiliam os pacientes e tem uma rotina assídua ao lado das pessoas infectadas é de suma importância que se avalie os efeitos da alta carga de trabalho devido a pandemia sobre a sua saúde mental, uma vez que necessita-se estar bem consigo para desenvolver um bom trabalho. Por isso, incentivos para uma condição 
melhor de trabalho, treinamento correto do uso de Equipamentos de Proteção Individual (EPI's) e, principalmente, atendimento voltado a saúde mental são de extrema importância para estes profissionais (BARBOSA DJ, et al., 2020).

Diante disso, o presente trabalho teve como objetivo fazer uma revisão de característica integrativa, apresentando uma síntese de resultados obtidos através de artigos na literatura acerca da saúde mental dos enfermeiros que atuam na linha de frente no combate ao COVID-19 nesse tempo de pandemia no Brasil e uma apresentação, também, sobre o que se faz para o incentivo destes profissionais e o auxílio quanto ao tratamento de sua saúde mental.

\section{MÉTODOS}

Trata-se de um estudo de Revisão Integrativa da Literatura (RIL) que, segundo Polit DF e Beck CT (2019), é uma pesquisa que visa gerar uma síntese da literatura ou identificar o estado da arte sobre determinado assunto ou o tema a ser investigado, bem como conhecer quais as lacunas sobre ele.

Para a condução do estudo, utilizou-se as diretrizes estabelecidas pelo Preferred Reporting Items for Systematic Reviews and Meta-Analyses (PRISMA) e o modelo de revisão de seis etapas: elaboração da pergunta norteadora; busca ou amostragem na literatura; coleta de dados; análise crítica dos estudos incluídos; discussão dos resultados; e apresentação da revisão integrativa (GALVÃO TF, et al., 2015; SOUZA MT, et al., 2010).

Como questão norteadora de pesquisa estabeleceu-se: "Qual o panorama da saúde mental da enfermagem durante a COVID-19 no Brasil?". Como guia para elaboração da pergunta norteadora desta revisão e a busca na literatura, foi utilizada a estratégia do acrônimo PICO (SANTOS CMC, et al., 2007), conforme apresentado na Quadro 1.

Quadro 1 - Elementos da estratégia PICO.

\begin{tabular}{|l|l|}
\hline \multicolumn{1}{|c|}{ Componente } & \multicolumn{1}{c|}{ Definição } \\
\hline P: População ou problema & Enfermagem brasileira \\
\hline I: Intervenção & Saúde mental na COVID-19 no Brasil \\
\hline C: Controle ou comparação & Nenhuma \\
\hline O: Resultados ou desfecho & Panorama da saúde mental da enfermagem \\
\hline
\end{tabular}

Fonte: Ferreira ACR e Barros FRB, 2021.

$\mathrm{Na}$ busca/amostragem pelas bases de dados, os cruzamentos foram realizados na forma de associação utilizando o operador booleano and e os Descritores em Ciências da Saúde (DECS) e seus correspondentes no Medical Subject Headings (MeSH): "COVID-19/COVID-19", "Saúde mental/Mental Health" e "Enfermagem/Nursing".

As bases de dados online consultadas, no período de fevereiro de 2020 a junho de 2021, foram: Banco de Dados em Enfermagem (BDENF) Latino-Americano e do Caribe de Informação em Ciências da Saúde (LILACS), National Library of Medicine (PUBMED) e Scientific Electronic Library Online (SCIELO).

Os artigos que compuseram o corpus de análise foram selecionados a partir dos seguintes critérios de inclusão: artigos publicados na íntegra online, no idioma português, inglês e/ou espanhol entre os anos de 2020 e 2021.

Foram excluídos os artigos em que não foi possível identificar relação com a temática por meio da leitura de título e resumo, estudos secundários, relato de caso, literatura cinzenta, reflexões e editoriais. Os artigos encontrados em mais de uma base de dados foram considerados somente uma vez. O processo de seleção dos artigos está apresentado na Figura 1. 
Figura 1 - Fluxograma da busca dos artigos da revisão.

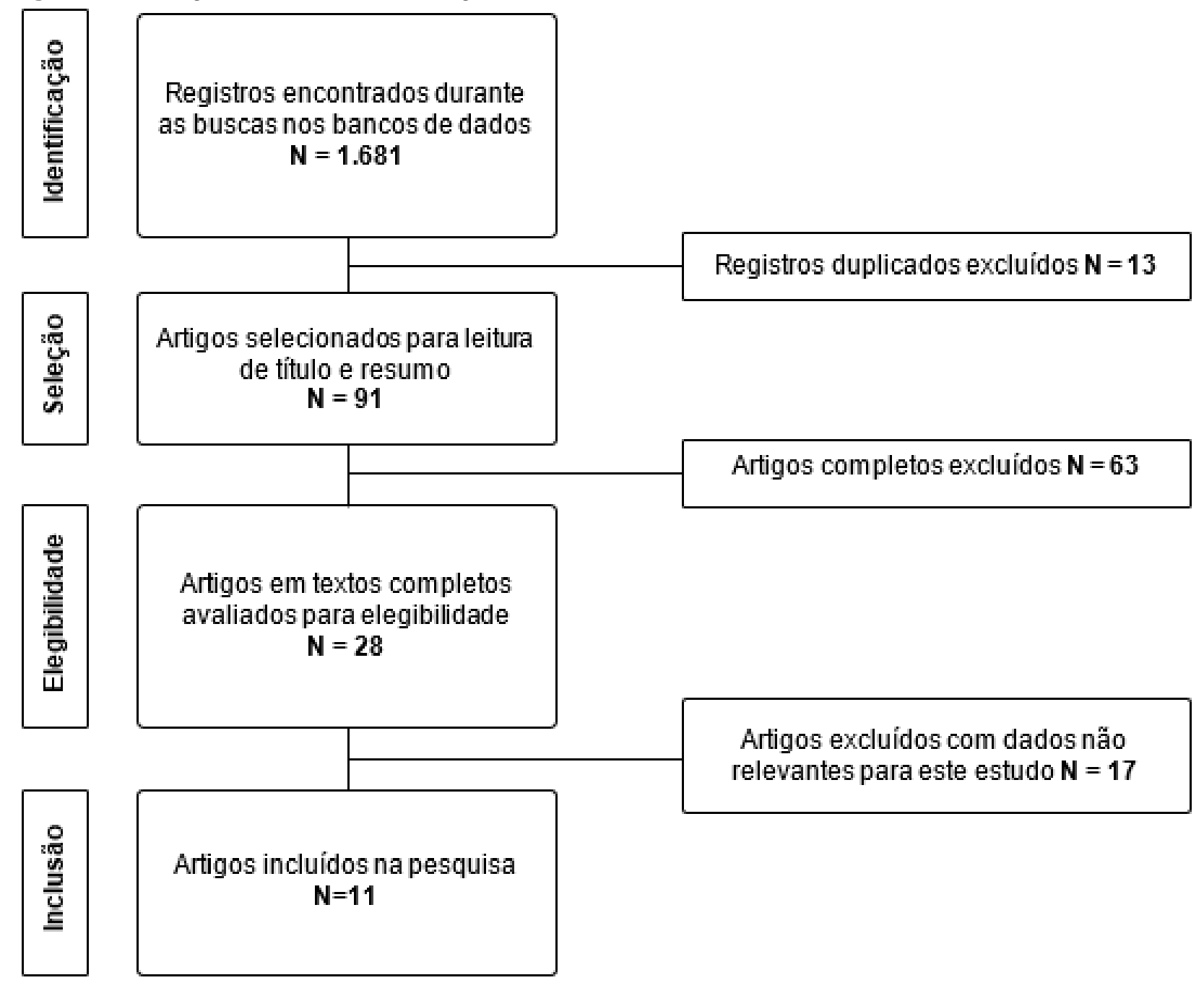

Fonte: Ferreira ACR e Barros FRB, 2021.

Para organização e tabulação dos dados dos estudos selecionados, se utilizou de um protocolo de revisão integrativa para esse fim. Nesse protocolo se fez necessário identificar o título do estudo, elaboração da pergunta norteadora, sua instituição-sede, o local em que o trabalho foi publicado, as características metodológicas da pesquisa e a avaliação do rigor metodológico (SOUZA MT, et al., 2010).

Os dados identificados nos estudos receberam análise crítica dos resultados por meio de leituras mais frequentes e reflexivas, permitindo a interpretação dos dados e posterior discussão através de frequências simples e percentuais em consonância com as variáveis estabelecidas previamente no instrumento de coleta de dados. Os resultados foram apresentados na forma de tabelas, sendo estes discutidos a luz da literatura.

\section{RESULTADOS}

A partir dos cruzamentos nas bases de dados estabelecidas, foram identificados um total de 1.681 artigos, sendo 19 artigos na base de dados BDENF, 25 artigos na LILACS, 1.200 artigos na PUBMED e 437 artigos na SCIELO. Foram selecionados 28 estudos para leitura da íntegra. Destes, 11 foram considerados elegíveis, mas 17 não apresentavam dados que respondessem à pergunta da pesquisa. Por fim, 11 artigos compuseram a amostra. O Quadro 2 apresenta as principais informações dos artigos incluídos e analisados nesta revisão. 
Quadro 2 - Estudos incluídos na revisão.

\begin{tabular}{|c|c|c|c|c|}
\hline Código & Título do artigo & Autores/Ano & Periódico & $\begin{array}{l}\text { Base de } \\
\text { Dados }\end{array}$ \\
\hline A1 & $\begin{array}{l}\text { Fatores de estresse nos profissionais } \\
\text { de enfermagem no combate à } \\
\text { pandemia da COVID-19: Síntese de } \\
\text { Evidências }\end{array}$ & $\begin{array}{l}\text { BARBOSA DJ, et } \\
\text { al., } 2020\end{array}$ & $\begin{array}{l}\text { Comunicação em } \\
\text { Ciências da Saúde: } \\
\text { Reports in Health } \\
\text { Science }\end{array}$ & LILACS \\
\hline $\mathrm{A} 2$ & $\begin{array}{l}\text { Nurse's protagonism in structuring } \\
\text { and managing a specific unit for } \\
\text { COVID-19 }\end{array}$ & $\begin{array}{l}\text { BITENCOURT JV, } \\
\text { et al., } 2020\end{array}$ & $\begin{array}{l}\text { Texto e Contexto } \\
\text { Enfermagem }\end{array}$ & SCIELO \\
\hline A3 & $\begin{array}{l}\text { A saúde mental da enfermagem no } \\
\text { enfrentamento da COVID- } 19 \text { em um } \\
\text { hospital universitário regional }\end{array}$ & $\begin{array}{l}\text { DAL'BOSCO EB, et } \\
\text { al., } 2020\end{array}$ & $\begin{array}{l}\text { Revista Brasileira } \\
\text { de Enfermagem }\end{array}$ & LILACS \\
\hline A4 & $\begin{array}{l}\text { Challenges faced by pediatric nursing } \\
\text { workers in the face of the COVID- } 19 \\
\text { pandemic }\end{array}$ & $\begin{array}{l}\text { GÓES FGB, et al., } \\
2020\end{array}$ & $\begin{array}{l}\text { Revista Latino- } \\
\text { Americana de } \\
\text { Enfermagem }\end{array}$ & PUBMED \\
\hline A5 & $\begin{array}{l}\text { Saúde mental dos profissionais de } \\
\text { enfermagem do Brasil no contexto da } \\
\text { pandemia COVID-19: Ação do } \\
\text { Conselho Federal de Enfermagem }\end{array}$ & $\begin{array}{l}\text { HUMEREZ DC, et } \\
\text { al., } 2020\end{array}$ & $\begin{array}{l}\text { Cogitare } \\
\text { Enfermagem }\end{array}$ & LILACS \\
\hline A6 & $\begin{array}{l}\text { Desafíos para la enfermeira de salud } \\
\text { mental después del COVID- } 19\end{array}$ & $\begin{array}{c}\text { MARTINEZ- } \\
\text { ESQUIVEL D, } 2020\end{array}$ & $\begin{array}{l}\text { Scientific Journal of } \\
\text { Nursing }\end{array}$ & LILACS \\
\hline A7 & $\begin{array}{l}\text { Presenteeism in nurses: comparative } \\
\text { study of Spanish, Portuguese and } \\
\text { Brazilian nurses }\end{array}$ & $\begin{array}{l}\text { MOSTEIRO-DÍAZ } \\
\text { MP, et al., } 2020\end{array}$ & $\begin{array}{l}\text { Internacional } \\
\text { Nursing Review }\end{array}$ & PUBMED \\
\hline A8 & $\begin{array}{l}\text { The health of helathcare } \\
\text { professionals coping with the COVID- } \\
19 \text { pandemic }\end{array}$ & $\begin{array}{l}\text { TEIXEIRA CF, et al. } \\
\qquad 2020\end{array}$ & $\begin{array}{l}\text { Ciência e Saúde } \\
\text { Coletiva }\end{array}$ & SCIELO \\
\hline A9 & $\begin{array}{l}\text { A infecção por coronavírus chegou ao } \\
\text { Brasil, e agora? Emoções de } \\
\text { enfermeiras e enfermeiros }\end{array}$ & $\begin{array}{l}\text { ELERES FB, et al., } \\
\qquad 2021\end{array}$ & $\begin{array}{l}\text { Revista Brasileira } \\
\text { de Enfermagem }\end{array}$ & SCIELO \\
\hline A10 & $\begin{array}{l}\text { Depressão e ansiedade em } \\
\text { profissionais de enfermagem durante } \\
\text { a pandemia da COVID-19 }\end{array}$ & $\begin{array}{l}\text { SANTOS KMR, et } \\
\text { al., } 2021\end{array}$ & Escola Anna Nery & SCIELO \\
\hline A11 & $\begin{array}{l}\text { Work environment of hospital nurses } \\
\text { during the COVID-19 pandemic in } \\
\text { Brazil }\end{array}$ & $\begin{array}{l}\text { SANTOS JLG, et } \\
\quad \text { al., } 2021\end{array}$ & $\begin{array}{l}\text { Internacional } \\
\text { Nursing Review }\end{array}$ & PUBMED \\
\hline
\end{tabular}

Fonte: Ferreira ACR e Barros FRB, 2021.

Dentre os 11 artigos (100,0\%) selecionados para o trabalho, 8 foram publicados no ano de 2020 (72,7\%) e 3 foram publicados no ano de 2021 (27,3\%). Não se buscou artigos relacionados aos anos anteriores, visto que como a produção diz respeito à pandemia da COVID-19, não faria sentido buscar em anos anteriores uma vez que a pandemia teve seu ápice, no Brasil, no ano de 2020.

De acordo com a revista em que os artigos selecionados se encontram, 5 desses artigos $(45,4 \%)$ foram encontrados em periódicos relacionados à Enfermagem e 6 destes (54,6\%) foram encontrados em periódicos sobre saúde geral e coletiva.

Acerca dos bancos de dados pesquisados, os resultados mostram que na plataforma LILACS foram encontrados 4 artigos relevantes (36,4\%), na plataforma SCIELO 4 artigos $(36,4 \%)$, na plataforma PUBMED foram encontrados 3 artigos $(27,2 \%)$ e na plataforma BDENF não foi encontrado nenhum artigo relevante $(0 \%)$, pois, todos os artigos que resultaram da busca se tratava de revisões ou reflexões, o que se enquadra nos critérios de exclusão. Para melhor elucidação quanto a abordagem de conteúdo de cada artigo, construiuse o Quadro 3, onde faz-se a apresentação dos artigos de acordo com as variáveis: objetivo, tipo de estudo e tipo de abordagem do estudo. 
Quadro 3 - Características dos estudos incluídos na revisão.

\begin{tabular}{|c|c|c|c|}
\hline Código & Objetivo & Tipo de Estudo & $\begin{array}{c}\text { Abordagem do } \\
\text { Estudo }\end{array}$ \\
\hline A1 & $\begin{array}{l}\text { Identificar os principais efeitos psicológicos da } \\
\text { pandemia da COVID-19 nos profissionais de } \\
\text { enfermagem; descrever os principais fatores capazes } \\
\text { de gerar estresse psicológico nos profissionais de } \\
\text { enfermagem; descrever as estratégias de coping para } \\
\text { o combate ao estresse emocional. }\end{array}$ & Qualitativo & Observacional \\
\hline $\mathrm{A} 2$ & $\begin{array}{l}\text { Relatar a experiência no processo de estruturação e } \\
\text { gestão de uma unidade específica para COVID-19, } \\
\text { ressaltando o protagonismo do enfermeiro nas } \\
\text { tomadas de decisão. }\end{array}$ & Misto & Observacional \\
\hline A3 & $\begin{array}{l}\text { ldentificar a prevalência e fatores associados à } \\
\text { ansiedade e depressão em profissionais de } \\
\text { enfermagem que atuam no enfrentamento da COVID- } \\
19 \text { em hospital universitário. }\end{array}$ & Misto & Transversal \\
\hline A4 & $\begin{array}{l}\text { Identificar os desafios que os trabalhadores de } \\
\text { enfermagem pediátrica enfrentam em decorrência da } \\
\text { pandemia COVID- } 19 \text {. }\end{array}$ & Qualitativo & Transversal \\
\hline A5 & $\begin{array}{l}\text { Refletir sobre a saúde mental dos profissionais de } \\
\text { enfermagem brasileiros no contexto da pandemia } \\
\text { COVID- } 19 \text {. }\end{array}$ & Qualitativo & Transversal \\
\hline A6 & $\begin{array}{l}\text { Descrever as realidades, avanços e desafios para a } \\
\text { enfermagem em saúde mental após a pandemia } \\
\text { COVID-19. }\end{array}$ & Qualitativo & Observacional \\
\hline A7 & $\begin{array}{l}\text { Comparar os níveis de presenteísmo em três amostras } \\
\text { de enfermeiras e identificar a relação entre o } \\
\text { presenteísmo e características sociodemográficas e } \\
\text { profissionais. }\end{array}$ & Misto & $\begin{array}{l}\text { Transversal/ } \\
\text { Multicêntrico }\end{array}$ \\
\hline A8 & $\begin{array}{l}\text { Sistematizar um conjunto de evidências científicas } \\
\text { apresentadas em artigos internacionais que identificam } \\
\text { os principais problemas que estão afetando os } \\
\text { profissionais de saúde envolvidos diretamente no } \\
\text { enfrentamento da pandemia de COVIS-19 e apontam } \\
\text { as estratégias para a proteção e a assistência à saúde } \\
\text { desses profissionais. }\end{array}$ & Misto & Observacional \\
\hline A9 & $\begin{array}{l}\text { Investigar as emoções de enfermeiras acerca da } \\
\text { infecção por coronavírus (COVID-19). }\end{array}$ & Qualitativo & Descritivo \\
\hline A10 & $\begin{array}{l}\text { Analisar a prevalência de sintomas depressão, } \\
\text { ansiedade e fatores associados em profissionais da } \\
\text { equipe de enfermagem durante a pandemia da COVID- } \\
\text { 19. }\end{array}$ & Misto & Transversal \\
\hline A11 & $\begin{array}{l}\text { Investigar o ambiente de trabalho dos enfermeiros em } \\
\text { hospitais universitários durante a pandemia COVID-19 } \\
\text { no Brasil. }\end{array}$ & Misto & $\begin{array}{l}\text { Transversal/ } \\
\text { Observacional }\end{array}$ \\
\hline
\end{tabular}

Fonte: Ferreira ACR e Barros FRB, 2021.

\section{DISCUSSÃO}

O SARS-CoV-2, vírus causador da COVID-19 apresentou seus primeiros casos no Brasil em meados de janeiro de 2020 (MINISTÉRIO DA SAÚDE, 2021). Desde a confirmação de infecções, o número de casos aumentou consideravelmente, tendo em vista que este vírus possui alta taxa de transmissão (CIOTTI M, et al., 2020). É de conhecimento geral que, no Brasil, esse surto de COVID-19 acabou causando um colapso geral nos hospitais ao redor do país, causando pânico na população e, principalmente, exigindo demasiado daqueles que precisavam trabalhar na linha de frente no combate ao vírus: os profissionais da saúde. 
Dentre os profissionais da saúde que trabalharam no combate ao vírus, podemos destacar os enfermeiros, pois são esses os profissionais responsáveis por permanecer, a maior parte do tempo ao lado de seus pacientes, guiando-os e os acalmando em meio ao caos vivido. Diante da incerteza sobre o novo vírus e a calamidade vivida pela superlotação de hospitais em todo o país, exigiu-se demais dos profissionais de enfermagem, onde estes tinham que trabalhar por horas seguidas em meio às incertezas e inseguranças (BARBOSA DJ, et al., 2020; DAL'BOSCO EB, et al., 2020).

Visando auxiliar os profissionais que estavam, e ainda estão atuando na linha de frente, o Conselho Federal de Enfermagem (COFEN) (2020) manteve-se ativo no que diz respeito ao treinamento e conhecimento dos enfermeiros acerca da pandemia de COVID-19, e apresentou suas preocupações com o uso adequado de EPI's, mantendo-se sempre atualizado sobre as normas apresentadas pela Organização Mundial da Saúde (OMS) e Ministério da Saúde (MS) (HUMEREZ DC, et al., 2020).

O presenteísmo destacou-se nos tempos de pandemia durante o trabalho de enfermeiros. Acerca do assunto, podemos citar o trabalho de Mosteiro-Díaz MP, et al. (2020), onde se avaliou esse sentimento entre os enfermeiros espanhóis, brasileiros e portugueses. No trabalho, cita-se que os enfermeiros brasileiros apresentam baixos níveis de presenteísmo e que enfermeiros mais jovens e com menos experiência profissional apresentam níveis mais baixos. O autor cita também a importância de treinamento prévio, principalmente a nível universitário, dos enfermeiros que vão para o mercado de trabalho. Com o devido treinamento e incentivo, os enfermeiros passam a apresentar menos quadros de transtornos mentais frente a estresses como o da pandemia de COVID-19.

Em seu cotidiano, os enfermeiros precisam lidar com sentimentos como sofrimentos, dor, morte e perdas, o que já acaba acarretando um quadro de estresse emocional (HUMEREZ DC, et al., 2020). Por esse motivo, o trabalho do enfermeiro já é considerado como um gerador de sofrimento psíquico, por causa das condições de trabalho à que são submetidos. Diante de todos os problemas enfrentados causados devido à pandemia, pouco se falou acerca da saúde mental dos enfermeiros que lutaram e lutam, até os dias atuais, na linha de frente no combate ao vírus (DUARTE MLC, et al., 2018).

Perante a situação de pandemia vivida, a OMS acredita que os enfermeiros estão mais susceptíveis a desenvolverem transtornos mentais, por conta do excesso de trabalho e das incertezas diante do novo vírus, como altos níveis de ansiedade, falta de sono causada por estresse e depressão (HUMEREZ DC, et al., 2020; ELERES FB, et al., 2021). Sabe-se também que os casos de Síndrome de Burnout aumentaram consideravelmente entre os enfermeiros que atuaram na linha de frente dos hospitais (HUMEREZ DC, et al., 2020).

No trabalho realizado no Hospital Filantrópico do Estado de Santa Catarina por Bitencourt JV, et al. (2020), os autores relataram como foram adquiridas medidas para o tratamento dos pacientes com COVID-19 de forma que isso pudesse, também, favorecer e auxiliar o trabalho dos profissionais enfermeiros envolvidos. Para tanto, a primeira medida tomada foi envolver todos os profissionais da saúde em reuniões sobre medidas de combate ao COVID-19, de forma a ouvi-los, principalmente os enfermeiros. Também, o hospital mostrou preocupação quanto ao equipamento de proteção individual utilizado pelos enfermeiros, dando cursos que auxiliavam os profissionais quanto à proteção.

Todas essas medidas adotadas pelo hospital mostraram aos profissionais enfermeiros uma maior confiança no combate ao vírus e no tratamento dos pacientes acometidos e, devido a essa confiança e apoio, os profissionais se sentiam mais seguros e apresentaram poucos quadros ou quadros mais amenos de transtornos mentais (BITENCOURT JV, et al., 2020).

Por outro lado, em um trabalho realizado por Dal'Bosco EB, et al. (2020) no Hospital Universitário Regional dos Campos Gerais (HURCG) da Universidade Estadual de Ponta Grossa, foi realizada a aplicação de questionários aos enfermeiros para tentar avaliar os índices de ansiedade e depressão entre os profissionais que atuaram na linha de frente. O HURCG é considerado um hospital de referência quando se fala no tratamento e combate à COVID-19. Na pesquisa, os autores destacam que a maior parte da amostra foi composta por mulheres, com mais de 40 anos e que houve, dentre os transtornos mentais, uma prevalência 
maior de ansiedade, apresentando $48,9 \%$ da amostra e, logo em seguida, depressão com $25 \%$ acometendo $25 \%$ das pessoas entrevistadas. Nesta pesquisa também, destacou-se que os enfermeiros atuavam com regime de 40 horas de trabalho semanal.

Resultados semelhantes aos expostos acima foram apresentados no trabalho de Santos JLG, et al. (2021), onde foi feita uma análise da prevalência de sintomas como depressão e ansiedade em profissionais enfermeiros que atuaram durante a pandemia. Assim como o estudo citado acima, a maioria dos entrevistados foram enfermeiras que trabalhavam em setor privado e foram apontados pela maioria das entrevistadas sintomas de transtornos como ansiedade e depressão muito prevalente. $\mathrm{O}$ autor ainda cita a importância de políticas que visem auxiliar o trabalho dos enfermeiros, principalmente o que incita a segurança durante 0 trabalho.

Acerca do ambiente de trabalho dos enfermeiros em hospitais universitários durante a pandemia de COVID-19, podemos citar o trabalho de Santos KMR, et al. (2021), onde se avaliou a qualidade do ambiente de trabalho oferecido aos profissionais de enfermagem durante a pandemia em três hospitais universitários no Brasil. Como resultado, verificou-se que a maioria dos hospitais ofereceu aos seus enfermeiros um treinamento acerca do uso de EPI's e que os profissionais entrevistados apresentaram um medo de ser infectado. Esse medo também se estende à infecção de familiares.

Eleres FB, et al. (2021) realizaram seu estudo em uma instituição de ensino superior privada localizada na cidade de Fortaleza, Ceará-BR, onde teve como objetivo principal investigar as emoções dos profissionais enfermeiros durante o enfrentamento da pandemia de COVID-19. Seus resultados mostraram que, os principais sentimentos apontados foram ansiedade, cansaço, medo, insegurança, angústia e dor emocional devido ao cenário de incertezas de conhecimento acerca do vírus. Porém, alguns enfermeiros apontaram, também, sentimentos como tranquilidade e bem-estar, apontando a sua preocupação com seus pacientes e com os familiares desses pacientes, esses sentimentos eram mencionados quando seus pacientes conseguiam superar a crise causada pelo vírus.

Em comparação com os estudos anteriores, é interessante apontar que, ao que tudo indica, os enfermeiros precisam principalmente de incentivo e apoio. Esse apoio dos órgãos públicos tem impacto sobre as suas emoções, onde os profissionais se sentem mais seguros para trabalhar, eles tendem a desenvolver pensamentos mais positivos, mesmo em meio a todo o caos vivido durante a pandemia (ELERES FB, et al., 2021).

Um estudo que ressalta o que foi dito acima é o de Humerez DC, et al. (2020), onde eles citam ações que o Conselho Federal de Enfermagem (COFEN) (2020) tomou e ações que podem tomar para auxiliar o trabalho dos enfermeiros durante a pandemia a fim de minimizar os efeitos quanto à saúde mental. Dentre os principais problemas que acabam somando para um quadro de piora na saúde mental dos enfermeiros, estão destacados a falta de treinamento correto para o enfrentamento da COVID-19, o que acaba acarretando o medo de, principalmente, contaminar familiares; é ressaltada também a baixa remuneração, o que acaba desmotivando os profissionais.

Porém, os autores apontam uma medida, dita como a mais importante, tomada pelo COFEN: a disposição de profissionais especializados em saúde mental para o tratamento de enfermeiros que estão na linha de frente. Essa oferta por tratamento especializado é uma das medidas mais importantes pois, com o tratamento correto e terapias, os profissionais podem tratar os medos e inseguranças que surgem devido ao trabalho excessivo (HUMEREZ DC, et al., 2020).

Está claro que, quanto mais incentivo os profissionais da linha de frente recebem, mais seguros se sentem para trabalhar, o que tende a diminuir os quadros de transtornos mentais desenvolvidos. Portanto, ações como a do COFEN, em investir no treinamento correto dos enfermeiros quanto ao uso de EPIs e, principalmente, a informação passada a esses profissionais, é de extrema importância para que eles se sintam seguros diante do caos vivido. Ainda, a oferta de terapias por especialistas em saúde mental é de extrema importância para que esses profissionais se sintam bem, não só fisicamente, como emocionalmente para cuidar de seus pacientes que também vivem tempos de incertezas e medos (HUMEREZ DC, et al., 2020). 


\section{CONSIDERAÇÕES FINAIS}

Desde o início da pandemia da COVID-19, os profissionais que mais trabalham na linha de frente são os profissionais da saúde. Dentre estes, podemos destacar os enfermeiros, que são os responsáveis por manter a boa relação entre pacientes e outros profissionais, são quem tem mais contato com esses pacientes e quem cabe acalmá-los nesses tempos de calamidade. Contudo, sendo os que mais têm responsabilidade, vem também o desgaste, tanto físico quanto emocional, que esses profissionais sofrem. Diante disso, é comum que por conta do medo, da insegurança, excesso de trabalho em tempos de pandemia, esses profissionais passem a desenvolver transtornos mentais. Acerca do assunto, ficou claro que quanto mais seguros esses profissionais se sentem, menos quadros de transtornos mentais são apresentados. Portanto, cabe as políticas públicas buscarem formas de treinar corretamente esses profissionais para lidar com cenários caóticos, como o da pandemia de COVID-19 pois, quanto mais seguros eles se sentem no ambiente de trabalho, menos quadros de transtornos mentais apresentam.

\section{REFERÊNCIAS}

1. BARBOSA DJ, et al. Fatores de estresse nos profissionais de enfermagem no combate à pandemia da COVID-19: Síntese de Evidências. Comunicação em Ciência da Saúde, 2020; 31(suppl 1): 31-47.

2. BITENCOURT JV, et al. Nurse's protagonism in structuring and managing a specific unit for covid-19. Texto e Contexto Enfermagem, 2020; 29: e20200213.

3. CIOTTI M, et al. The COVID-19 pandemic. Critical Reviews in Clinical Laboratory Sciences, 2020; 57(6): 365-388.

4. CONSELHO FEDERAL DE ENFERMAGEM (COFEN). Resolução COFEN no 634, 26 de março de 2020. Autoriza e normatiza a teleconsulta de enfermagem como forma de combate à pandemia provocada pelo novo coronavírus (Sars-Cov-2). Brasil, 2020. Disponível em: http://www.cofen.gov.br/resolucao-cofen-no-0634-2020_78344.html. Acesso em: 10 jul. 2021.

5. DAL'BOSCO EB, et al. A saúde mental da enfermagem no enfrentamento da COVID-19 em um hospital universitário regional. Revista Brasileira de Enfermagem, 2020; 73(suppl 2): e20200434.

6. DUARTE MLC, et al. O trabalho em emergência hospitalar: sofrimento e estratégias defensivas dos enfermeiros. Revista Gaúcha de Enfermagem, 2018; 39: e2017-0255.

7. ELERES FB, et al. A infecção por coronavírus chegou ao Brasil, e agora? Emoções de enfermeiras e enfermeiros. Revista Brasileira de Enfermagem, 2021; 74(suppl 1): e20201154.

8. GALVÂO TF, et al. Principais itens para relatar revisões sistemáticas e meta-análises. Epidemiol Serv Saúde, 2015; 24(2): 335-342.

9. GOES FGB, et al. Challenges faced by pediatric nursing workers in the face of the covid-19 pandemic. Revista LatinoAmericana de Enfermagem, 2020; 28: e3367.

10. GORBALENYA AE, et al. The species Severe acute respiratory syndrome-related coronavirus: classifying 2019-nCoV and naming it SARS-CoV-2. Nature Microbiology, 2020; 5: 536-544.

11. HUANG C, et al. Clinical features of patients infected with 2019 novel coronavirus in Wuhan, China. The Lancet, 2020; 395: 497-506.

12. HUMEREZ DC, et al. Saúde mental dos profissionais de enfermagem do Brasil no contexto da pandemia Covid-19: ação do Conselho Federal de Enfermagem. Cogitare Enfermagem, 2020; 25: e74115.

13. LAl J, et al. Factors associated with mental health outcomes among health care workers exposed to coronavirus disease 2019. JAMA Network Open, 2020; 3(3): e203976.

14. MARTINEZ-ESQUIVEL D. Desafíos para la enfermería de salud mental después del COVID-19. Rev Cienc Cuidad., $2020 ; 17(3): 122-129$.

15. MINISTÉRIO DA SAÚDE. Boletim Epidemiológico 52 - Doença pelo Coronavírus COVID-19. Brasil, 2021. Disponível em: https://www.gov.br/saude/pt-br/media/pdf/2021/marco/05/boletim_epidemiologico_covid_52_final2.pdf. Acesso em: 10 jul. 2021.

16. MOSTEIRO-DÍAZ MP, et al. Presenteeism in nurses: comparative study of Spanish, Portuguese and Brazilian nurses. International Nursing Review, 2020; 67(4): 466-475.

17. PRADO $A D$, et al. $A$ saúde mental dos profissionais de saúde frente à pandemia do COVID-19: uma revisão integrativa. Revista Eletrônica Acervo Saúde, 2020; e4128.

18. SANTOS CM, et al. A estratégia PICO para a construção da pergunta de pesquisa e busca de evidências. Rev Latinoam Enfermagem, 2007; 15(3): 1-4.

19. SANTOS JLG, et al. Work environment of hospital nurses during the COVID-19 pandemic in Brazil. International Nursing Review, 2021; 68(2): 228-237.

20. SANTOS KMR, et al. Depressão e ansiedade em profissionais da enfermagem. Escola Anna Nery, 2021; 25(spe): e20200370.

21. SHEREEN MA, et al. COVID-19 infection: Origin, transmission, and characteristics of human coronaviruses. Journal of Advanced Research, 2020; 24: 91-98.

22. SOUZA MT, et al. Revisão integrativa: o que é e como fazer. Einstein, 2010; 8(1): 102-106.

23. TEIXEIRA CF, et al. The health of healthcare professionals coping with the covid-19 pandemic. Ciencia e Saude Coletiva, 2020; 25(9): 3465-3474.

24. WANG D, et al. Clinical Characteristics of 138 Hospitalized Patients with 2019 Novel Coronavirus-Infected Pneumonia in Wuhan, China. JAMA, 2020; 323(11): 1061-1069.

25. WORLD HEALTH ORGANIZATION (WHO). Novel coronavirus (COVID-19). 2020. Disponível em: https://www. who.int/emergencies/diseases/novel-coronavirus-2019. Acesso em: 31 ago. 2021

26. ZHU N, et al. A Novel Coronavirus from Patients with Pneumonia in China, 2019. New England Journal of Medicine, 2020; 382: 727-733. 